\title{
Depresión y drogodependencia: efectos sobre la salud dental
}

\section{Depression and drug dependency: effects on dental health}

\author{
Jiménez Polanco MP*, Astudillo Jiménez $\mathrm{O}^{* *}$, Mata Brotons $\mathrm{V}^{* *}$, Jorge Ferre Jorge \\ J**, Correia d' Oliveira NG**
}

\section{RESUMEN}

Introducción: Los pacientes que reciben antidepresivos y los pacientes drogodependientes presentan xerostomía, lo que junto a la alteración de sus hábitos higiénicos lleva a un incremento en la aparición de caries dental. Este trabajo compara dos muestras de ambos grupos.

Material y métodos: Se han tomado datos de 75 pacientes en tratamiento antidepresivo y 80 pacientes drogodependientes durante un periodo de cinco años, en el servicio de psiquiatría del Hospital Clínic Universitari de Barcelona. Los datos recogidos han sido número y gravedad de caries, piezas dentales ausentes y $\mathrm{pH}$ salival. Se han realizado las pruebas $U$ de Mann-Whitney, $t$ de Student y análisis de la varianza con el programa SPSS v. 11.5.

Resultados: Con un intervalo de confianza del $99 \%$ se establece que el grupo de pacientes drogodependientes presenta más caries y más graves, así como un pH más ácido que el grupo de pacientes con antidepresivos , aunque presentan menor ausencia de piezas dentales.

Discusión: Nuestros resultados son similares a los publicados en diversas áreas geográficas en lo que respecta a la aparición de caries en estos grupos. En nuestra muestra la pertenencia al grupo de drogodependientes tiene más peso que factores como el pH salival, el sexo o la edad. En la literatura se intenta discernir el peso que la patología de base, la higiene oral o la propia medicación tienen sobre la salud dental, pero es difícil llegar a conclusiones determinantes.

Palabras clave: Caries antidepresivos, metadona, drogodependencia, estudio descriptivo transversal.

\section{SUMMARY}

Introduction: Patients receiving antidepressants and drug dependent patients present xerostomy, which together with the alteration of their hygienic habits leads to an increase in the occurrence of dental caries. This study compares two samples of both groups.

Materials and methods: Data were collected from 75 patients in antidepressant treatment and 80 drug addict patients during a period of five years in the psychiatric department of the Hospital Clínic Universitari from Barcelona. The data collected were number and severity of caries, number of missing teeth and salivary $\mathrm{pH}$. Mann-Whitney $U$ test, Student's $t$ test and analysis of variance were performed using SPSS v. 11.5.

Results: Drug dependent patients present more caries and are more serious, and have a more acidic $\mathrm{pH}$ than the group of patients receiving antidepressants, but have less absence of teeth. This was established with a confidence interval of $99 \%$.

Discussion: Our results regarding caries in these groups are similar to those reported in different geographical areas. In our sample, being drug dependent has more weight than factors such as salivary $\mathrm{pH}$, sex or age. Literature try to discern the weight that underlying pathology, oral hygiene or medication have on dental health, but it is difficult to obtain decisive conclusions.

* Directora del Postgrado de Estética en Odontología. Universidad de Barcelona.

** Departamento de Odontología Estética y Conservadora. Facultad de Odontología. Universidad de Barcelona. 
Key words: Caries, antidepressants, methadone, drug addiction, descriptive sectional study.

Fecha de recepción: 1 de octubre 2009.

Aceptado para publicación: 23 de octubre 2009.

Jiménez Polanco MP, Astudillo Jiménez O, Mata Brotons V, Jorge Ferre Jorge J, Correia d' Oliveira NG. Depresión y drogodependencia: efectos sobre la salud dental Av. Odontoestomatol 2011; 27 (1): 41-46.

\section{INTRODUCCIÓN}

El riesgo de sufrir la enfermedad denominada caries es multifactorial. Se considera una enfermedad de origen bacteriano de desarrollo lento causada por $S$. Mutans y Lactobacillus. Como en todo proceso infeccioso importa la agresividad del agente causal y la resistencia del huésped. En el caso de la caries la resistencia viene dada por la calidad del esmalte, la cantidad y calidad de la saliva y unos correctos hábitos higiénicos y dietéticos.

Son numerosos los factores que pueden disminuir el flujo salival: situaciones fisiológicas con efecto anticolinérgico, medicamentos $(1,2)$, enfermedades de las glándulas salivales o procesos sistémicos que las afecten $(3,4)$.

Entre los fármacos que inducen la hiposialia, tanto los antidepresivos como la metadona tienen un destacado papel. Además, las enfermedades en cuyo tratamiento son utilizados también incluyen factores de riesgo para la hiposialia y la mala higiene oral $(5,6)$.

Aproximadamente un $6 \%$ de la población norteamericana puede llegar a tomar antidepresivos y el $63 \%$ de los pacientes que toman antidepresivos tricíclicos pueden quejarse de sensación de boca seca (7). Los antidepresivos disminuyen la secreción salival y, como cada vez se recetan más antidepresivos en asistencia primaria, estos efectos se ven cada vez más (8).

De hecho, uno de cada cinco pacientes que van al dentista expresan síntomas de depresión y la propia depresión está asociada con disminución de flujo salival, sequedad oral y alteración del gusto (9). Con la hiposalivación disminuye la concentración de bicarbonato, calcio, fosfatos y proteínas en la saliva y aumenta el recuento de lactobacillus (10).
No todos los antidepresivos tienen el mismo efecto sobre el flujo salival. La amitriptilina, maprotilina y clomipramina tienen un efecto pronunciado. La nortriptilina produce una inhibición del $56 \%$ de la producción de saliva total no estimulada, la fenoxetina del $34 \%$ y la mianserina del $29 \%$. La zimedilina (200 mg/día) no tiene más efecto anticolinérgico que imipramina ( $75 \mathrm{mg} /$ día). Por último, citalopram, flupenthixol, isocarboxazida y citrato de litio no tienen efecto sobre la salivación (11-13).

En la encuesta nacional de 1987 un 0,6 \% de la población española era consumidora habitual de heroína, descendiendo su consumo a un 0,3\% en 1996. Las encuestas también reflejan una poliadicción. De los usuarios de heroína, el 100 \% consumen cannabis y cocaína, un 91 \% otros opiáceos y un $80 \%$ tranquilizantes $(14,15)$.

Es sabido que los drogadictos tienen poco cuidado de su salud en general y tienen trastornos de conducta e infecciones asociadas a la drogadicción (16), igualmente tienen alta incidencia de caries y enfermedad periodontal (17).

Los consumidores de metanfetamina tienen caries rampantes por el efecto xerostómico, incremento del consumo de azúcar y negligencia en su cuidado. Acuden a la consulta dental sólo cuando se presenta dolor y no cumplen los tratamientos prescritos (18). Por su parte, los consumidores de cannabis sufren sus efectos sobre la cavidad oral: aumento de la incidencia de carcinoma de células escamosas, xerostomía, gingivitis severa, necrosis isquémica del paladar y bruxismo (19). El tratamiento con metadona no está exento de efectos secundarios para los dientes puesto que produce xerostomía (20). Además, los pacientes con metadona presentan un aumento en el consumo de azúcar (21). 
El presente trabajo pretende contribuir al conocimiento sobre los efectos que estas dos patologías (depresión y drogodependencia) y sus tratamientos, habituales en un servicio hospitalario de psiquiatría, tienen sobre la salud dental.

\section{MATERIAL Y MÉTODOS}

Se ha estudiado una muestra de 155 pacientes (75 varones y 80 mujeres) con un rango de edad de 15 a 88 años que corresponde a dos grupos, pacientes con tratamiento antidepresivo o drogodependientes en tratamiento en el servicio de psiquiatría del Hospital Clínic de Barcelona entre los años 2000-2005.

El grupo de pacientes en tratamiento antidepresivo está formado por 30 varones y 45 mujeres con un rango de edad de 15 a 88 años y el grupo de pacientes drogodependientes está formado por 45 varones y 35 mujeres con un rango de edad de 17 a 47 años (Tabla 1). En ambos grupos se han estudiado las variables $\mathrm{pH}$ salival, medido mediante tiras reactivas (Merck), número de piezas dentales ausentes, número de piezas dentales con caries y el grado máximo de gravedad de caries que presentaba el paciente (Tabla 2).

El análisis estadístico se ha realizado con el programa SPSS v. 11.5 para Windows. Se establece un nivel de confianza estadístico de un 99\% para el presente trabajo. Para comparar la gravedad máxima de caries se ha realizado la prueba $U$ de MannWhitney y para el resto de comparaciones se ha realizado la $t$ de Student. Se ha realizado una prueba ANOVA para discernir qué factores determinan el número de piezas con caries.

\begin{tabular}{|l|c|c|}
\hline \multicolumn{3}{|c|}{ TABLA 1.- DESCRIPTIVA DE LOS } \\
GRUPOS DE PACIENTES \\
\hline & Antidepresivos & $\begin{array}{c}\text { Drogo- } \\
\text { dependientes }\end{array}$ \\
\hline $\mathrm{n}$ & 75 & 80 \\
Hombres [n (\%)] & $30(40)$ & $45(56,3)$ \\
Edad (rango) & $15-88$ & $17-47$ \\
Edad (media: & & \\
desviación estándar) & $43,9(16,9)$ & $29,3(6)$ \\
\hline
\end{tabular}

TABLA 2.- GRADOS EN LA GRAVEDAD DE CARIES

\begin{tabular}{|c|c|}
\hline 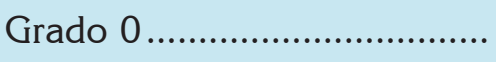 & No caries \\
\hline & Ise 1 y 111 \\
\hline & $\mathrm{Ca}$ \\
\hline & Gra \\
\hline
\end{tabular}

\section{RESULTADOS}

Los pacientes drogodependientes presentan mayor gravedad de caries $(99,01$ frente a 53,64$)$ y más caries $(12,8$ frente a 7,8$)$ que los pacientes en tratamiento antidepresivo. Igualmente el grupo de pacientes drogodependientes presenta un $\mathrm{pH}$ más ácido $(6,8$ frente a 7,4$)$ y menor ausencia de piezas dentales $(3,3$ frente a 6,8$)$ que el grupo de pacientes en tratamiento antidepresivo, pudiendo este hecho atribuirse a la mayor juventud de los primeros respecto a los segundos (Tabla 3 ).

TABLA 3.- COMPARATIVA DE LOS DOS GRUPOS DE PACIENTES

\begin{tabular}{|c|c|c|c|}
\hline & $\begin{array}{l}\text { Antidepresivos } \\
\qquad n=75\end{array}$ & $\begin{array}{l}\text { Drogodependientes } \\
\qquad n=80\end{array}$ & $\mathbf{P}$ \\
\hline Gravedad caries (rango promedio) & 53,64 & 99,01 & $<0,01^{a}$ \\
\hline Piezas con caries (media) .... & 7,8 & 12,8 & $<0,01^{b}$ \\
\hline $\mathrm{pH}$ (media) & 7,4 & 6,8 & $<0,01^{\mathrm{b}}$ \\
\hline 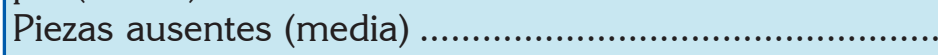 & 6,8 & 3,3 & $<0,01^{\text {b }}$ \\
\hline Edad (media). & 43,9 & 29,3 & $<0,01^{b}$ \\
\hline
\end{tabular}

${ }^{a}:$ U de Mann-Whitney; ${ }^{b}$ : $\mathrm{t}$ de Student. 
El análisis de la varianza muestra que el número de piezas con caries depende de la pertenencia al grupo de pacientes drogodependientes y no de otras variables como edad, sexo o $\mathrm{pH}$ intraoral.

\section{DISCUSIÓN}

Nuestros resultados en lo que respecta a la prevalencia de caries en un grupo de drogadictos son similares a otros estudios realizados en España (22), Italia (23), Dinamarca (24), Estados Unidos (25), Francia (26) o Australia (17). Scheutz ha mostrado que un grupo de drogadictos necesitaba una media de 1,7 obturaciones nuevas cada año (24). Los resultados de Reece en un estudio transversal de 280 individuos entre 19 y 45 años, divididos en un grupo de drogadictos y otro que no lo era, mostró que el grupo de adictos presentaba más caries y ausencias dentarias que el grupo de no adictos. En el grupo de adictos la patología se desarrolla en edad más joven. Entre los menores de 35 años el 56,8 \% de los adictos presentaba patología frente a un 5,4\% de los no adictos (27). Esto es consistente con nuestros resultados, en el que el grupo de drogodependientes presenta mayor cantidad y gravedad de caries que quienes no habían sido drogodependientes.

A pesar de que el índice de caries aumenta con la edad, según un estudio de Kumar con 220 pacientes psiquiátricos institucionalizados (28), en nuestro trabajo el factor edad no es predictivo, importando mucho más el hecho de ser drogodependiente. A pesar de ser un grupo con una media de edad significativamente menor, parece ser que su falta de cuidado general y oral en particular, pesa más que el efecto xerostómico en el grupo consumidor de antidepresivos, aceptando incluso que este grupo puede tener también cierto descuido personal.

Un trabajo clarificador respecto a la relación entre hiposialia y cuidado personal en un grupo de pacientes con antidepresivos fue el realizado por Rindal, que estudió 915 individuos con un mínimo de 55 años y 4 años de medicación. Observó que un grupo cuya única medicación xerostomizante eran los antidepresivos presentaba mayor número de restauraciones a lo largo de los cuatro años que un grupo sin medicación alguna, pero que no presentaba diferen- cias significativas respecto a un grupo con medicación no xerostomizante. Es decir, no parece ser tanto la hiposialia como la falta de cuidado personal lo que incrementa la cantidad de caries, pues el estudio asumía que el grupo sin medicación tenía un cuidado general y oral mejor que el medicado. Este trabajo mostraba, además, los efectos a largo plazo sobre la salud oral de cierto tratamiento antidepresivo (29). Esto coincide con el trabajo de Rundegren con un grupo de 20 pacientes con tratamiento antidepresivo durante 5 años, en el que el $55 \%$ de los pacientes presentaba nuevas caries en dicho periodo, mostrando la necesidad de un programa de profilaxis (30).

En nuestro trabajo, el pH del grupo drogodependiente es significativamente menor que el del grupo con antidepresivos, aunque el análisis de la varianza no le otorga un valor predictivo. Hemos visto la morbilidad sobre la salud dental de dos patologías tratadas en los servicios de psiquiatría que afectan a dos grupos de edad diferentes aunque ambas en aumento. Los problemas de drogadicción y sus necesidades de rehabilitación se incrementan en la juventud y por otra parte, tal como indica Chapman (31), la depresión será, tras la enfermedad cardíaca, la segunda causa de perdida de calidad de vida en edades avanzadas. Es importante, por ello, incorporar pautas de prevención de la patología oral derivada de estas enfermedades y sus tratamientos.

\section{BIBLIOGRAFÍA}

1. Guggenheimer J, Moore PA. Xerostomia: etiology, recognition and treatment. J Am Dent Assoc 2003;134:61-9.

2. Smith RG, Burtner AP. Oral side-effects of the most frequently prescribed drugs. Spec Care Dentist 1994;14:96-102.

3. Formiga F, Mascaró J, Vidaller A, Pujol R. Xerostomía en el paciente anciano. Rev Mult Gerodontol 2003;13:24-8.

4. Rodríguez-Landa JF, Contreras CM. Algunos datos recientes sobre la fisiopatología de los trastornos por ansiedad. Rev Biomed 1998;9:18191. 
5. Friedlander AH, Friedlander IK, Gallas M, Velasco E. Late-life depression: its oral health significance.Int Dent J 2003;53:41-50.

6. Antilla SS, Knuuttila ML, Sakki TK. Depressive symptoms as an underlying factor of the sensation of dry mouth. Psychosom Med 1998;60:215-8.

7. Hunter KD, Wilson WS. The effects of antidepressant drugs on salivary flow and content of sodium and potassium ions in human parotid saliva. Arch Oral Biol 1995;40:983-9.

8. Peeters FP, de Vries MW, Vissink A. Risks for oral health with the use of antidepressants. Gen Hosp. Psychiatry 1998;20:150-4.

9. D'Mello DA. Are your patient depressed? Implications for dental practice. J Mich Dent Assoc 2003;85:26-32.

10. Bardow A, Nyvad B , Nauntofte B. Relationships between medication intake, complaints of dry mouth, salivary flow rate and compositions, and the rate of tooth demineralization in situ. Arch Oral Biol 2001;46:413-23.

11. Von Knorring L. Changes in saliva secretion and accomodation width during short-term administration of imipramine and zimelidine in healthy volunteers. Int Pharmacopsychiatry 1981; 16:69-78.

12. Clemmesen L, Jensen E, Min SK, Bolwing TG, Rafaelsen OJ. Salivation after single-doses of the new antidepressants femoxetine, mianserin and citalopram. A cross-over study. Pharmacopsychiatry 1984; 17:126-32.

13. Rafaelsen OJ, Clemmesen L, Lund H, Mikkelsen PL, Bolwing TG. Comparison of peripheral anticholinergic effects of antidepressants: dry mouth. Acta Psychiatr Scand (suppl) 1981;290: 364-9.

14. Navarro Botella A, Gómez Gonzalez E. La incidencia de las drogas en el mundo laboral en la Comunidad de Madrid. Madrid: Cance; 1998: 255-88.
15. CIDUR-EDIS. Juventud y drogas en España. Dirección General de la Juventud. Madrid: Ministerio de Cultura; 1980.

16. Titsas A, Ferguson MM. Impact of opioid use on dentistry. Aust Dent J 2002;47:94-8.

17. Carter EF. Dental implications of narcotic addiction. Aust Dent J 1978;23:308-10.

18. Shaner JW. Caries associated with methanphetamine abuse. J Mich Dent Assoc 2002;84: 42-7.

19. Fazzi M, Vescovi P, Savi A, Manfredi M, Peracchia $M$. The effects of drugs on the oral cavity. Minerva Stomatol 1999;48:485-92.

20. Graham $\mathrm{CH}$, Meecham JG. Dental management of patients taking methadone. Dent Update 2005;32:477-8.

21. Zador D, Lyons Wall PM, Webster J. High sugar intake in a group of women on methadone maintenance in south western Sydney- Australia. Addiction 1996;91:1053-61.

22. Rodríguez Vázquez C, Cabello ML, Quijada E, Riobóo R. Evaluación de la salud oral en una población de drogodependientes. Av Odontoestomatol 2002; 18:153-60.

23. Angelillo IF, Grasso GM, Sagliocco G, Villari P, D'Errico MM. Dental health in a group of drug addicts in Italy. Community Dent Oral Epidemol 1991;19:36-7.

24. Scheutz F. Five-year evaluation of a dental care delivery system for drug addicts in Denmark. Community Det Oral Epidemiol 1984;12:2934.

25. Rosenstein DI, Stewart A. Dental care for patients receiving methadone. J Am Dent Assoc 1974;89: 356-9.

26. Bernardini AM, Camus JP. Etat buccodentaire chez le toxicomanie. Rev Odontoestomatol 1983; 12:169-73. 
27. Reece AS. Dentition of addiction in Queensland: poor dental status and major contributing drugs. Aust Dent J 2007;52:144-9.

28. Kumar M, Chandu GN, Shafiulla MD. Oral health status and treatment needs in institutionalized psychiatric patients: one year descriptive cross sectional study. Indian J dent Res 2006;17: 171-7.

29. Rindal DB, Rush WA, Peters D, Maupomé G. Antidepressant xerogenic medications and restoration rates. Community Dent Oral Epidemiol 2005;33:74-80.
30. Rundegren J, van Dijken J, Mörnstad H, von Knorring L. Oral conditions in patients receiving long-term treatment with cyclic antidepressant drugs. Swed Dent J 1985;9:55-64.

31. Chapman DP, Perry GS. Depression as a major component of public health for older adults. Prev Chronic Dis 2008;5:A22.

\section{CORRESPONDENCIA}

M Piedad Jiménez Polanco

E-mail: pjimenezestetica@ub.edu 